\title{
The Use of TINA Principles in the Management of Internet Multimedia Conferences
}

\author{
Q. Cabell, P. Kirstein, T. Pagtzis, T. Tiropanis and L. Wang \\ Department of Computer Science, University College London \\ Gower Street, London WC1E 6BT, United Kingdom \\ \{q.cabell, p.kirstein, t.pagtzis, t.tiropanis, 1.wang\}@cs.ucl.ac.uk
}

\begin{abstract}
This paper shows how some of the TINA principles used in the development of Service Management techniques for the Broadband ISDN may be applied to the management of Internet Services. In the original B-ISDN concept of these services, it was assumed that there would be B-ISDN components in both the network and subscriber systems. In the Internet version of the services, it was considered that IP Multicast would be used for the multimedia conferencing services. In the approach adopted here, the normal Internet functionality is maintained unchanged in the subscriber equipment; modifications in the access network dealt with the differences between the BISDN and the Internet. The paper discusses the architecture adopted, the applications services integrated and the methods employed to demonstrate remotely the system. The latter encompassed the novel use of Direct Broadcast Satellite connectivity, for the purposes of the demonstration.
\end{abstract}

\section{Introduction, Background and Motivation}

As a result of global deregulation and the development of new technologies, there is a huge growth in the aspirations of the Telecommunications Carriers as to the types of services they might offer in the future. There is a large body of research devoted to standardising the types of components that might be needed to provide these services, and the mechanisms that might be used for their interconnection.

A number of independent industry bodies (e.g. TINA Consortium, ITU-T) have sought to define standard architectures for the control and management of these telecommunications services. There has been a concerted effort to define open, reusable components for service control and service management in a common Service Architecture (TINA-SA) [16]. In particular, the TINA consortium has attempted to specify a service architecture [16] addressing the key areas of service management (subscription, accounting, profile management) and service control (setting up and controlling the exchanges amongst service users). This has been used as the basis for further investigation into service management and service control by a number of European projects like PROSPECT [6], and VITALv2 [20]. The last of these references was produced as part of the VITAL project; this is supported by the ACTS programme of the European Commission, and is validating the concepts of the TINA architectures. 
The VITAL project has developed an extensive architecture, and a number of reusable components. These include both network and application components. They collaborate using the CORBA communication primitives [4]. All the components developed under VITAL use these mechanisms - including the applications. The particular focus of most of the VITAL activity was with ATM networks - which are the proposed bearer for the European B-ISDN.

We decided to pursue a complementary approach in the light of the VITAL project effort. We wished to explore to what extent the TINA service management recommendations could be used to manage and control Internet-based applications. The use of TINA for the management of TCP IP-based networks has already been investigated and it has been shown that the TINA Network Resource Architecture [14] can provide for the management of TCP-IP networks [5] as well as B-ISDN networks. However, our focus is maintained in the pursuit to demonstrate a synergy between TINA and IP Multicast services. Therefore, we make the assumption that the Internet-workstations are unaware of TINA. In this way, no TINA components need reside on the workstations of the Internet users, deviating from the TINA prescriptions.

Our considerations departed from the standpoint that many enterprises were currently using Internet applications and that these would probably continue to be of interest whatever the shape of the future B-ISDN. Moreover, the Internet applications were already sophisticated and optimised to the Internet environment. For these reasons, we wished to work without changing the applications themselves - or even putting any special code in the User workstations as the TINA SA requires.

The fundamental applications that have presented particular research interest have been multi-point, multimedia conferencing, using IP Multicast to deal with the multiple participants [8]. Unfortunately, these applications did not match well either the TINA architecture or the component implementations. While the TINA architecture is considering how to embrace multicast, those aspects are still some distance off. In both architectural (TINA), and implementation (VITAL) viewpoints, TINA-oriented applications are point-point, and are initiated by a transmitter; on the contrary, multicast conferencing is normally announced through multicasting, while it is the receivers who decide to participate. From that it becomes clear that it is essential to consider variants of the standard multicast conferencing scenario to achieve a synergy between the TINA architecture and Internet multimedia conferencing applications. Several such variants were provided by the MERCI and MECCANO projects [8], and could be adopted for our TINA-Internet system synergy.

Many organisations and IP networks do not yet support multicast IP. To cater for such users, we had already developed, in the MERCI project, a full-duplex IPMulticast/IP-Unicast transcoder, called the UCL Transcoding Gateway (UTG) [18]. The UTG has a number of features not directly relevant to VITAL (like the ability to transcode), but its functionality was much more akin to the way VITAL expected user-streams to be controlled. In addition, while it was customary to announce 
multimedia conferences using a Session Announcement Protocol (SAP) [10], there is also a provision for inviting one or more people to such conferences using the Session Initiation Protocol (SIP) [12]. The use of the SIP mechanisms of initiating sessions between users and the UTG mechanisms for streaming user media content to/from Unicast hosts, matched well with the VITAL architecture implementation but also applied to Internet networks.

Section 2 presents a description on the components of the TINA architecture available through VITAL during the TINA-Internet synergy demonstration. Section 3, elaborates on the MERCI/MECCANO Internet components utilised in the system implementation, and considers how they had to be modified. Section 4 describes integration considerations between TINA and Internet as well as how the TINA components had to be modified or extended; Section 5 describes the Convergence- 1 DBS satellite receiver system. In Section 6 we describe the demonstrated synergy between TINA and Internet, through our integrated implementation. Finally, Section 7, brings together some conclusions, and reflects on the lessons learnt from such activity.

It should be noted here that during this last stage of the whole VITAL project effort, the particular research activity investigating the potentials for a synergy between TINA and the Internet, was kept deliberately small, for the timescale available. It was considered more appropriate to concentrate on the realisation of the idea of a synergistic environment between TINA and Internet, rather than producing a full-blown system completely from scratch. For that reason it was decided to utilise most of the TINA-core components already available in the VITAL project as well as use existing IP/IP Multicast applications that could approach the concept in question in a feasible and viable manner. Due to the complexity involved in establishing such synergy between TINA and Internet, it was deemed necessary to rule out further investigation of issues such as QoS, performance and scalability. Thus, despite the fact that such issues may be empirically addressed (albeit dimly) in the remaining sections of the paper, they are beyond the scope of this paper, and should be considered as basis for further work.

\section{The Supporting VITAL Architecture}

The existing VITAL Open Distributed Telecommunications Architecture (ODTA) is mainly based on the Telecommunications Information Networking Architecture (TINA) [15]. It expands, complements, and refines the TINA deliverables, as demanded by the VITAL implementation of this architecture to support the selected operational and management service features. The VITAL ODTA has been decomposed in several subsystems. These ODTA subsystems consist of Computational Objects (COs) according to TINA. These ODTA components consist of a set of interfaces with the corresponding operations. These interfaces are defined in the Interface Definition Language (IDL), as standardised by the Object Management Group (OMG) [3]. The ODTA components developed are distributed units. They run on top of a Distributed Processing Environment (DPE) installed in the terminals and in computing nodes within the retailer and connectivity provider 
domains. The DPE employed in VITAL is CORBA2.0 [4]. The DPE kernels are interconnected by means of a kernel transport network over TCP/IP. Most of the VITAL implementation runs over ATM.

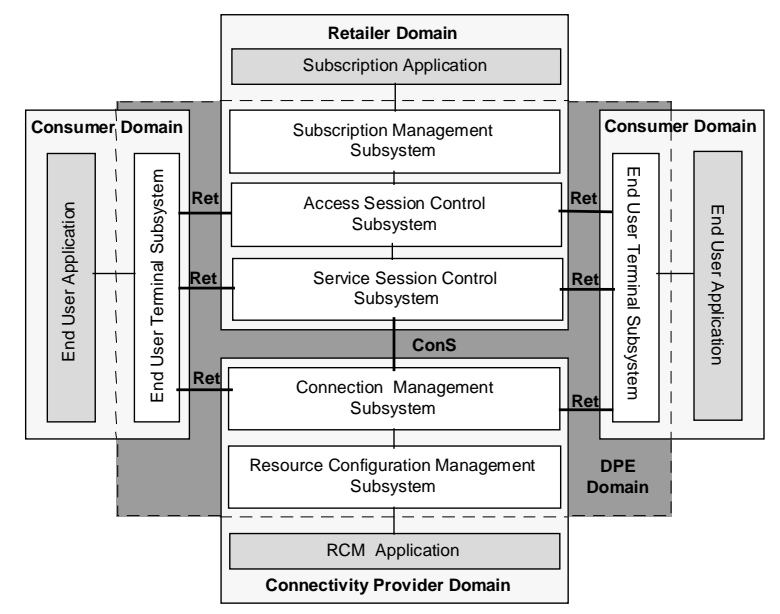

Fig. 1. Schematic Overview of the VITAL Architecture

The VITAL ODTA consists of components in the Consumer domain, and the Service Provider environment, as depicted in Fig. 1. The Provider environment consists of Retailer and Connectivity Provider domains. In VITAL, there are two types of terminals: end-user terminals and operator terminals. The end-user terminals in the Consumer domain entail the end-user applications (EA) and a set of components to interface with the Retailer domain. Among other EAs the Consumer domain included video-on-demand and other tele-training components [20]. The normal end-user terminals had to contain TINA-specific code, and be connected to the Retailer domain by the DPE. For the interaction between the subscribers and the service domains, a number of specific management components were added. These included a subscription management application (SA), which allows the interaction with the retailer, and a resource configuration management application (CA) for the connectivity provider.

The ODTA components are grouped in the following subsystems: End-User Terminal Subsystem (EU), Access Session Control Subsystem (AS), Session Control Subsystem (SC), Subscription Management Subsystem (SM), Connection Management Subsystem (CM), and Resource Configuration Management Subsystem (RC). Several TINA reference points were supported: the Ret-RP between the consumer domain and the retailer domain, and the ConS-RP between the retailer domain and the connectivity provider domain. Other reference points were also supported, but are beyond the scope of this paper. 


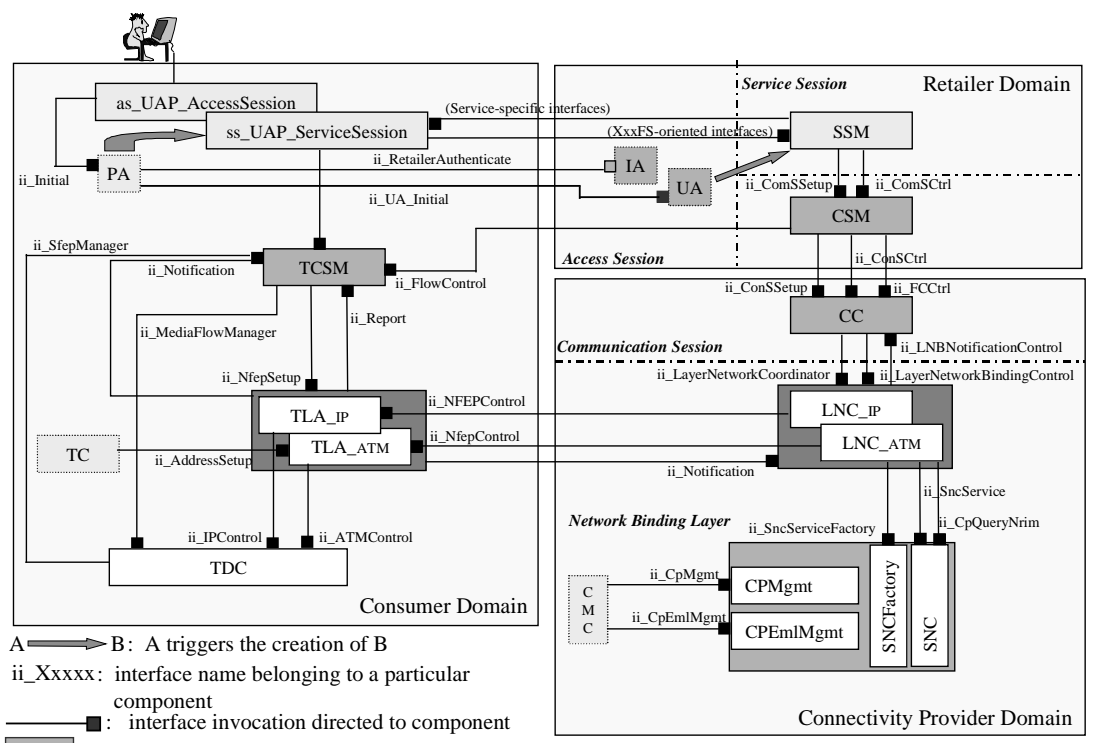

Yxxxx : TINA component

Fig. 2. Diagram of the VITAL-TINA component architecture

The core components of the VITAL architecture are located in the Retailer domain, as it may be seen from Fig. 1. These are Access Session and Service Session Control as well as Connection Management Subsystems. The Subscription Management and Resource Configuration Management Subsystems are really ancillary system services that complement the functions of the Retailer domain. They are structured in a layered approach being adherent to the Session Model prescribed by TINA [16], allowing retailers/service providers to deploy compound services independent of the underlying network. Fig. 2 provides a more focused view of the TINA-core components. This is only a partial view of the total component space in VITAL, but it includes the ones relevant towards the Internet synergy.

In the traditional TINA reference model, [14], the components participating in the Consumer Domain are located on the consumer's terminal and are an integral part in the communication activities of the DPE with the Retailer and further with the Connectivity Provider Domain. In the instance that the consumer wishes to make use of a service [21], it is essential that he/she gain access to a range of services by means of a Provider Agent (PA) through the normal login process (password authentication). Upon service selection, the service request is directed over the DPE, to the topmost layer of the Session model prescribed by TINA [16] - the Service Session Layer. There the Service Session Manager (SSM) in the Retailer Domain, will process the service request, before proceeding to the next stage - the setup of the Communication Session between the requester, the service and the available participants. The SSM 
may also undertake ancillary functions such as subscription and billing management; thus the service request is subject to validation under these functions prior to any further processing.

The setup of the Communication Session, is assigned on the next layer of the VITAL architecture, the Communication Session Layer. The CSM component aggregates from the participant consumer terminals the available range of what is called Stream Flow End Points (SFEPs). An SFEP represents a discrete characteristic of an available resource on the consumer's terminal. The range of SFEPs from each terminal is filtered and matched by the Connection Controller (CC) before submitted in a format that is recognised by the Network Controller (LNC). The latter establishes the connections between consumer terminals in relation to the physical network.

In the majority of the VITAL service, the DPE and the ODTA COs have run over ATM networks. Sessions between consumer terminals in the existing service implementations of VITAL are normally between single pairs of consumers.

\section{The MERCI/MECCANO Components}

The operation of the MERCI/MECCANO components is quite different from that of TINA through the VITAL DPE. Under the standard operational scenario, the network layer uses IP Multicast [13]. This implies that an IP Multicast tree is set up inside the network linking all the end points. The network backbone that encompasses such multicast capabilities is called collectively Multicast Backbone or M-Bone [7].

The applications under consideration are multimedia conferencing. Here each of the participants wishes to collaborate with all the others, using a combination of video, audio and shared workspace. In the normal operation, a conference is announced, by a broadcasting a Session Announcement, using Session Announcement Protocol (SAP), [10], on a particular multicast group with specific media characteristics. If a user wants to join a conference, it may send a JOIN request to a particular port on a specific multicast group. This then sends a JOIN request to the nearest active point part of the multicast tree; the appropriate branch is then set up to include the participant. An alternative to the above, is that one person may Invite another participant to the conference using the Session Initiation Protocol (SIP) [12]. The SIP data includes the same information as is provided in a SAP announcement. In either case the invitation is to a multicast conference. A schematic of the architecture of Internet multicast multimedia conferences is shown in Fig. 3. 


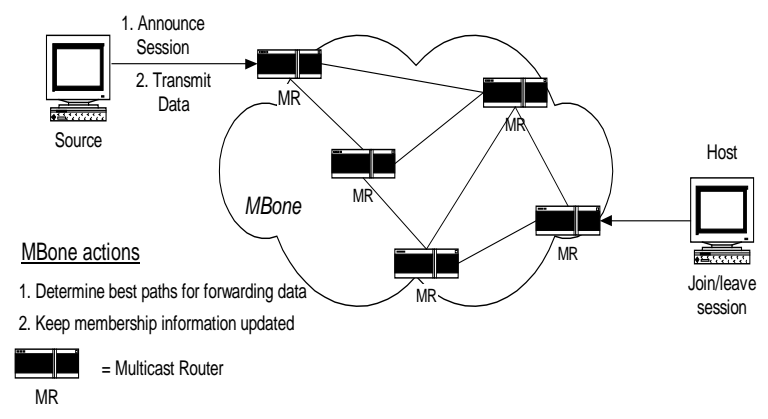

Fig. 3. Diagram of Multicast Conference Announcement and Operation

The above setup differs from the VITAL architecture in certain respects. The ODTA component implementation does not really envisage multicast, multi-person applications. Furthermore, the VITAL approach encourages any end-user activity to be managed in the Provider domain to facilitate session and communications management. However, by considering SIP (or SAP) which tackles multicast sessions it is possible to interface to the TINA-prescribed approach of session management in the current VITAL implementation.

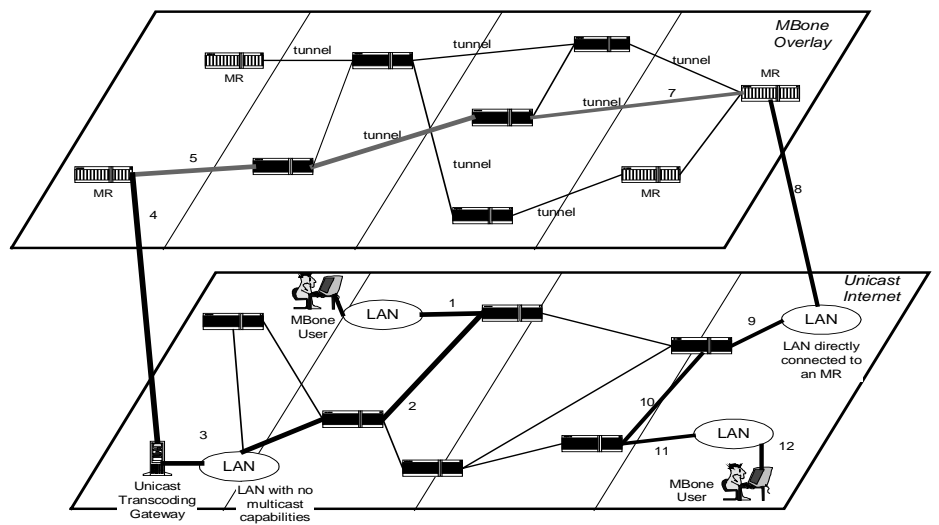

Fig. 4. Schematic of the UCL Transcoding Gateway

The issue of IP Multicast support by end-user hosts needs also to be addressed. It is often that not all organisations support IP Multicast. Also, the quality of network access may differ from one host to another. For this reason we developed, under MERCI/MECCANO, a Unicast Transcoding Gateway (UTG), with some interesting and relevant properties [17]. Fig. 4 provides an illustration of how UTG complements multicasting on a Unicast network. In particular, the UTG allows termination of multicast into unicast streams. At the same time the Trancoder takes the different sets of audio streams and multiplexes them onto the Unicast audio streams; it may also 
filter a number of multicast video streams activated for a particular conference session onto the Unicast one, while it passes through reliably any shared workspace streams.

\section{The Integrated TINA-Internet System}

The underlying idea for the integration of the TINA architecture and the Internet applications into a synergistic system, lies in the application of Service Control as well as Network Resource Allocation and Management onto new Internet services that exist at the moment unregulated. A characteristic instance of such modern Internet services is multimedia conferences over IP Multicast. In the light of the above, we have investigated extensions or restrictions of the existing set of the TINA Stakeholder Domains, as implemented in VITALv3, with respect to their coherence with the Internet world as depicted in Fig. 5.

Experiences from the VITAL-TINA architecture have made clear that the consumers' terminals, the instances of the Consumer Domain, contain normally a significant number of components to enable servicing by the Retailer Domain (through the mechanisms of DPE). In the Internet such view of the Consumer domain is largely discouraged - if not prohibited, since it leads to quite restrictive network designs in terms of management, scalability and performance. Such issues become more apparent as the number of available Internet services increases, yielding more complex management of the network, potential performance degradation, and the next to impossible task of managing terminal configurations to keep users online; all these need be accomplished in the context of heterogeneous systems networking.

In the Internet fashion, the Consumer Domain is populated by workstations connected directly to the Mbone, employing the lightweight mechanisms of Section 3. Such mode of operation precludes at the moment the opportunity for session management, billing or other services. However, since such core ancillary services become essential to the task of realising a synergy between TINA and Internet, we considered alleviating the complexity of the Consumer Domain component set by introducing an intermediate Access Network Domain (see Fig.5). The Consumer Domain is left simply as the bare configuration of workstations and Internet applications. The TINA-specific components of the simplified Consumer Domain are drawn to the extent they are required into the intervening Access Network Domain. In this manner, the Access Network Domain, would deal through its instances, with the specific aspects of interaction between Internet user workstations, monitored by a Core Network Domain. Fig. 5 presents an overview of our approach to the integration architecture. 


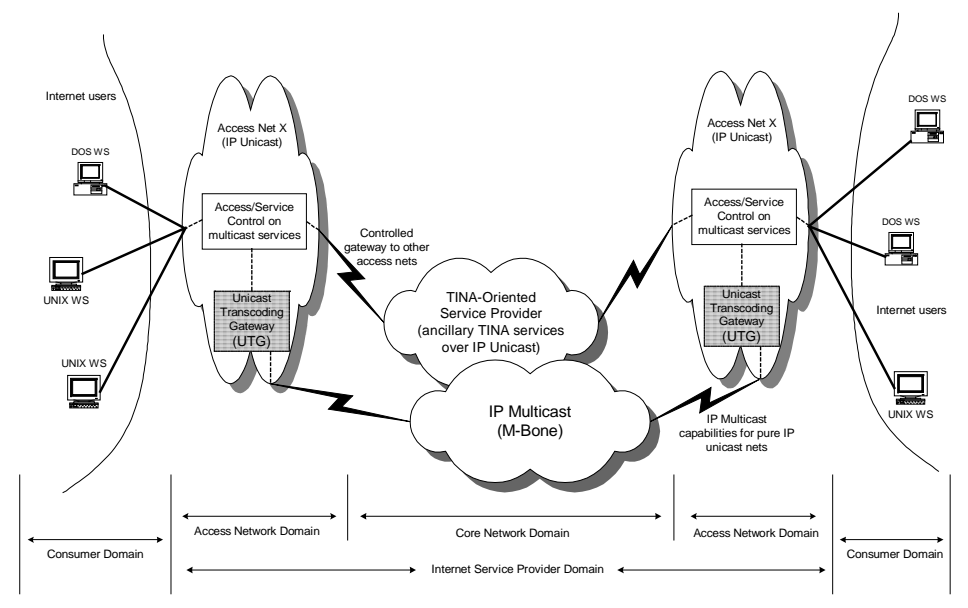

Fig. 5. Overview of the proposed synergistic architecture

Thus, for the integration of TINA components in managing Internet multicast conferences, we slightly extend the TINA architecture and map its domains to the Internet ones. The Retailer and Connectivity Provider Domains are selected to map collectively to the broader Internet Service Provider Domain. The Internet Service Provider Domain manages in turn, the Access Network and Core Network Domains. The Consumer Domain is simplified to be the user workstations (Internet users) with no TINA-specific functionality.

The fundamental idea under the concept of an Access Network is that both IP terminals and applications would access the core network via Access Points (APs) unique for each user. The Access Network would manage such APs. The latter would be closely coupled to the TINA components of the VITAL Retailer and Connectivity Provider Domain. The IP terminals need not be aware of TINA. In fact they could use the existing IP protocols to participate in service and communication sessions as they do today. Therefore, under such setup TINA becomes transparent to IP terminals.

Furthermore, the control of TINA stops at the Access Points. Our implementation proved it is not necessary for a provider to have control on the terminals of the endusers. The end-users can access the TINA services via access points. TINA and in particular its Service Session ancillary services can be applied efficiently by controlling these Access Points and not the terminals.

As a result of the decisions made above, there does have to be modification to some components of Fig. 2; these are restricted to those brought in the Access Network Domain. In particular, the Access Point Communications Session Manager (AP-TCSM), implemented (but reduced) the TCSM interfaces that the NRA requires [14]. Furthermore, the Access Point Layer Adapter (AP-TLA), implemented (but simplified) the TLA interfaces as prescribed by the TINA Network Resource Architecture. Last but certainly not least, the Access Point Application Adapter (APAA) implemented all the core functionality that the TINA Service Architecture 
required with respect to the Service Session Layer. In the VITALv3 the TINA implementation entailed such core functionality arranged in three components: ssUAP, TC and TDC. It was decided to leave out the functionality contained in TC and TDC as such components are directly related with the physical terminal activation or characteristics. The reason for that was twofold; first the TC and TDC functionality under the Internet paradigm is implicitly dissipated by the Internet applications; second the Access Network Domain does not maintain user terminals but accessibility to user workstations. The interactions among these components and the Provider domain are illustrated in Fig. 6.

Fig. 6. Schematic of the UCL TINA-Internet Architecture

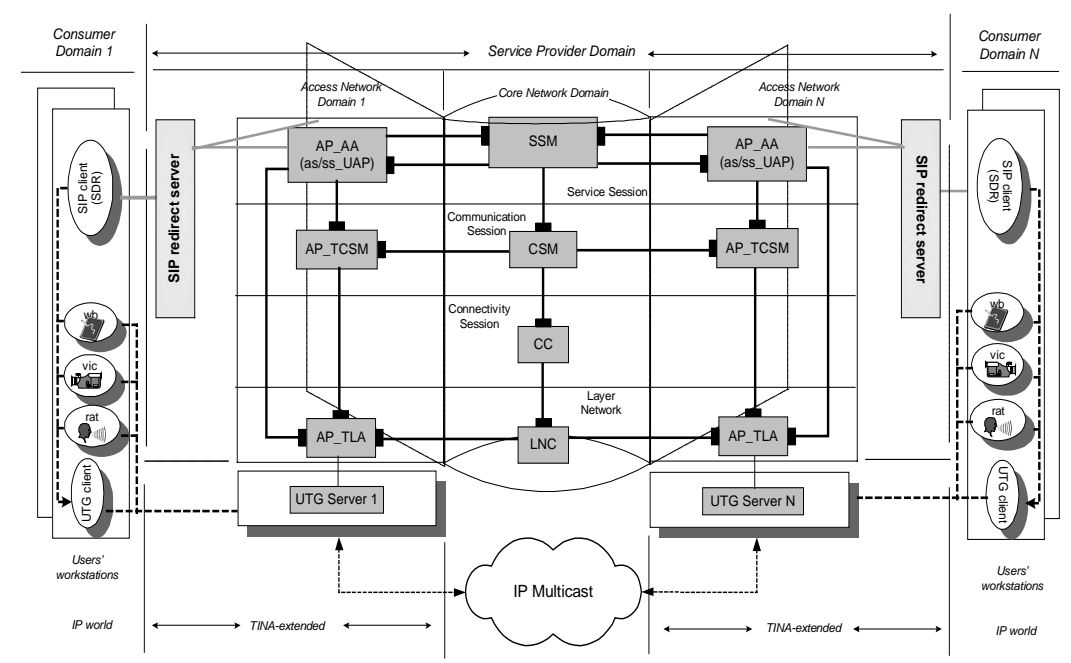

It should be noted that while Fig. 6 is drawn as if there were only two Access Network Domains, and two Consumer Domains, the system could scale well to as many as one requires. It is merely necessary to have one instance of the full Access Network Domain per network segment of user workstations. It is this version of Fig. 6 that has been implemented in the VITAL-Internet activity. This scales equally well with the TINA core components since they would be concerned only with Session management and not the actual network connections.

With respect to the Internet users of Fig. 5 and 6, we used workstations identical with the ones of Section 3. For interacting with the integrated TINA-Internet system we use these in their Unicast mode, and have them equipped with the UTG client and the multimedia tools for audio, video and shared workspace. Interaction between workstations was chosen to be primarily SIP [12]; that would allow users to invite other parties for participation in multimedia conferences. Such aligning decisions brought the system's design nearer the model prescribed by TINA in the VITAL access and service component implementations.

The communications interface between the user workstations and the Access Network Domain is managed by the latter through a SIP proxy server; this server 
would relay invitations to and from the user workstations. According to the SIP specification [12] this SIP proxy is following the behaviour of a SIP redirect server. Such server would be also empowered with the task of carrying out the appropriate local management functions (e.g. registering the User), while inviting other users to join the conferences from the same or - most importantly - different Access Network Domains.

With regard to the Core Network Domain shown in Fig. 6 its component set from the Service Session and up to the Communication Session Layer is largely unchanged from that of Section 2. However, on the Layer Network, there is a different communication technology employed for the purposes of the integrated system; this is the Internet (IP Unicast layer) in contrast to the normal VITAL underlying ATM communications transport. This renders the ATM-specific components, of the Connectivity Provider (see Fig.2), redundant for the purposes of our system. In VITAL, other partners have shown how TINA can control TCP/IP networks [5]. Departing from similar implementations the components for IP Unicast remain much the same as in the core VITALv3 components.

It may be noted that the VITAL implementation of TINA Network Resource Architecture does not consider normally the Connectivity Provider as part of an Access Network Domain. However, the potential of considering it in the light of the Internet paradigm, yields an elegant solution which simplifies as well as allows consistent and scalable management of an momentous task found in the topmost layers of the Core Network Domain: the task of Service Session Control. In fact the concept of TINA's Service Session Control may well constitute the enabling factor to have Internet applications integrated with "classical" telecom service applications.

\section{The Convergence-1 Presentation System}

Several difficulties were encountered during the task of getting adequate Internet facilities into the remote demonstration site. However, parallel work on the COIAS project [1], enabled the use of quasi-commercial satellite system called Convergence1. This system uses a DBS satellite, is operated by Easynet with BT, and is targeted at domestic end-stations. The earth-station equipment was developed by Eutelsat.

The Convergence-1 setup requires that the Receiving Workstation must be connected both to a switched network port (telephone modem or ISDN) and a domestic satellite receiver. The workstation must contact the ISP running the system (Easynet), via the switched access, but the incoming media streams may come via the satellite. The media streams to the up-link may come in via the Internet, and the switched return path must come in directly to Easynet, but may then be directed to anywhere else on the Internet. The direct connection is needed because the Easynet access control mechanism requires that the IP number of the receiving satellite machine to be part of the same network as that providing access control to the transmitter. Moreover, the usage assumed is either fully broadcast or Unicast. In the former case, it is not possible to address individual multicast groups; in the latter only one receiver can be addressed. The downlinks are operating at 2 MBPS, and thus the 
system is suitable for use with remote users. A schematic of the system is shown in Fig. 7.

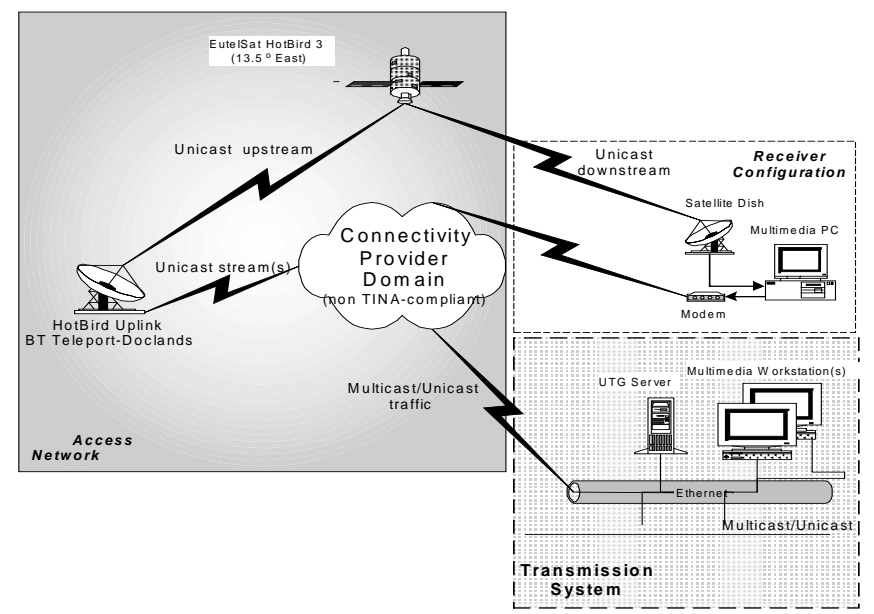

Fig. 7. Schematic of DBS configuration

When the satellite system acts in a Unicast way, then there must be a component near the access network which multiplexes the audio, aggregates the video and data, and transforms all IP streams into Unicast ones before transmitting the data through the access network to the satellite. This is the function of the UTG of the Section 3.

\section{The VITAL-Internet Synergy Demonstration}

For the VITAL Final Review, we set up a complete system based on the schematic of Fig. 6 and 7. We ran a total of eight machines, with the following functions:

At the UCL site:

- 2 Unix Machines acting as the Consumer Domains; these ran the full multimedia Mbone tools of VIC (packet video tool) [19], RAT (packet audio tool) [9], WB (shared editing) [22], SDR (Session Directory) [11], and the UTG Client (unicast mode) [18];

- One pair of Unix machines running through CORBA DPE the components of the Access Network Domain (SIP redirect server, AP_AA, AP_TCSM, AP_TLA) and the UTG Server;

- A second pair of Unix machines representing the second Access Network under identical component configuration as the first one;

- One Unix machine running the TINA core components with respect to Access and Service session control;

- 1 Satellite PC sited to monitor the streams seen in the demonstration site in Antwerp; this PC was attached to the PSTN. It communicated on the backchannel, via Easynet, with one of the UTGs (Unicast side); 
At the demo site (Antwerp):

- 1 Satellite PC to receive the media streams from the satellite. It communicated with Easynet via to the PSTN, and hence to the other UTGs (Unicast side);

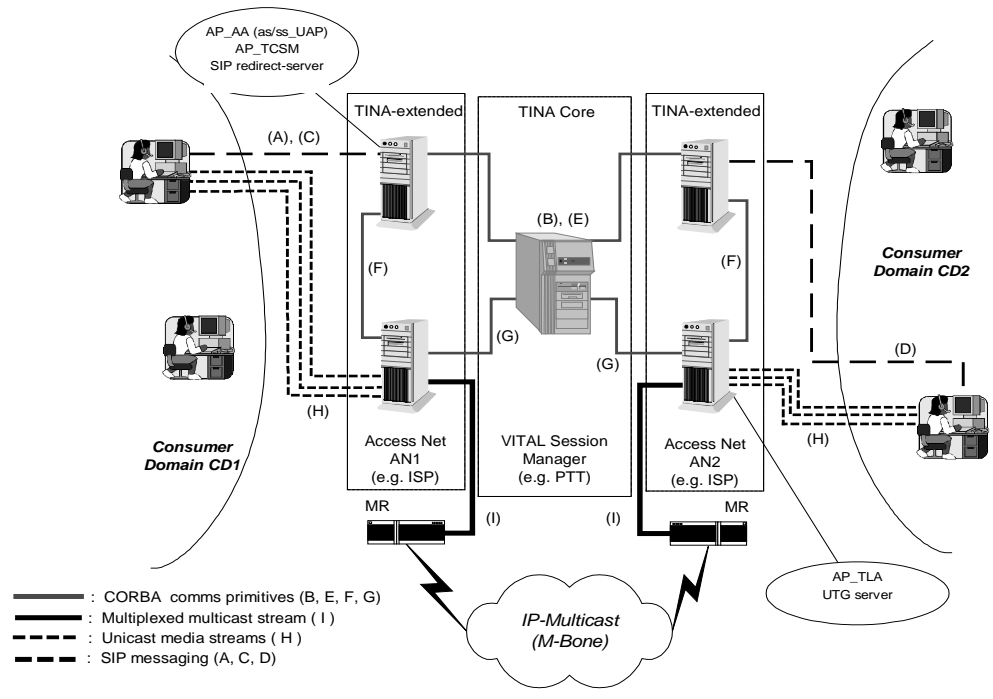

Fig. 8. Schematic of the Experimental Configuration

The Antwerp PC provided audio feedback; this was the best that could be achieved with the minimal modem back channel. The Layered Network technology used in both the Access Nets and the VITAL core net was only an Ethernet to communicate with the Consumer Domains through the VITAL service components. The full combination of Internet, Easynet and DBS satellite system was used to bring the receiving satellite PCs - both in Alcatel and UCL sites - in operation with respect to monitoring the demonstrated multimedia conference content. Their setup is presented in Fig. 9.

The demonstration scenario involved a somewhat contrived tele-tutoring session between a student and a tutor. Each of the two users belongs to a different Consumer Domain (CD): the student to $\mathrm{CD} 1$ and the tutor to $\mathrm{CD} 2$. These two users are assumed to reside in disparate network locations that were brought together by means of two different Access Networks. Fig. 8 depicts the two Access Networks as AN1 and AN2 respectively. The first of the two machines of AN1 and AN2 entailed the SIP redirect server, AP_AA and AP_TCSM components; the second machine was dedicated to the lower network layers by running the AP_TLA and the UTG server. Both machines per Access Net were closely coupled by means of CORBA communication primitives under the Access and Service control of the TINA core network machine.

Each user workstation (both tutor and student in this scenario) employed a set of Internet multimedia tools developed by various institutions. In particular, each user's 
machine had installed and configured VIC, RAT, WB and SDR as well as an enhanced version of the UTG client. VIC is a Unicast and multicast videoconferencing tool, developed by the Lawrence Berkeley Labs [19]. VIC can be used either for point-to-point videoconferencing involving a direct link between two hosts or for multiparty conferencing with many participants via the Internet Mbone. Both RAT [9] and WB [22] share similar connectivity properties with VIC, while they are used for audio conference and shared workspace sessions respectively. SDR (Multicast Session Directory) [11] is a tool which assists the user in setting up and joining conference announcements or invitations between two (or more) parties. RAT, WB and SDR were developed by UCL-CS.

Having started the operation of all machines in AN1, AN2 and the TINA core net, the tele-tutoring session was initiated by having the student (CD1) and tutor (CD2) to obtain access through their respective Access Nets (AN1, AN2). Such access request would be processed by each ANx and then sent to the TINA core for user logon registration. The most effective way of obtaining such access through SIP was by means of having each user send a SIP invitation to him/herself on the local Access Net. Response to the access request would be propagated back to the originator of the request, subject to password validation by both the Access Net and the TINA core. Upon successful user password validation, the TINA core would create an Access Session record for the logged user, with the subscription manager informed accordingly.

Upon successful access logon, both student and tutor may invite one another, by sending a SIP invitation at their local $\mathrm{ANx}$, destined to the other. Who actually initiates the invitation to the other does not matter. In the event where the student wants to invite the tutor for a session, the SIP invitation would travel from CD1 to the local AN1. There it would be processed and forwarded to the TINA core, which in turn would create a Service Session record for the particular originator of the request. The TINA core would also inform the relevant Access Session record of the invitation recipient. This would instruct the relevant TINA core components to dispatch the invitation to the appropriate ANx (AN2) where it would be managed accordingly and dispatched further to its local SIP redirect server for sending to the recipient.

It may be pointed out that the TINA core was structured so that the invitation could be stored in the event that it could not be delivered at that time to a remote ANx. However, there was an agreed time interval for which the invitation would be kept stored in the TINA core before the former was discarded. In that sense the TINA core would not support persistent delivery of invitations unless the time interval was set to infinite, which could prove rather ineffective in the event of excessive undelivered invitations.

At receipt of the invitation, the invitee may choose to accept or decline it. The response would be received from the local SIP server and sent through its ANx (AN2) back to TINA core and from there sent back to the ANx of the invitation originator (AN1). In the event of a decline response, the invitation originator would simply be informed of the decline. No change would occur in the respective access nets or the 
TINA core net, as the originator may wanted to place another invitation to another user.

In the event of an accept response the originator's ANx (AN1) would proceed to setup the communication and network layers on both itself and indirectly the remote ANx (AN2) by means of alerting the respective layers of the TINA core. The latter would ultimately trigger the network layer components of each ANx to instruct their local UTG servers to connect specific ports for transcoding between each local UTG server and the user workstation from the local consumer domain for each medium (Audio, Video and Whiteboard). The output of the transcoding would be destined through the appropriate network elements (multicast enabled routers) on the M-Bone. Since both UTG servers are connected to the M-Bone, stream connections would be established between the workstations of both the student and the tutor for all three mediums and the two would be in session.

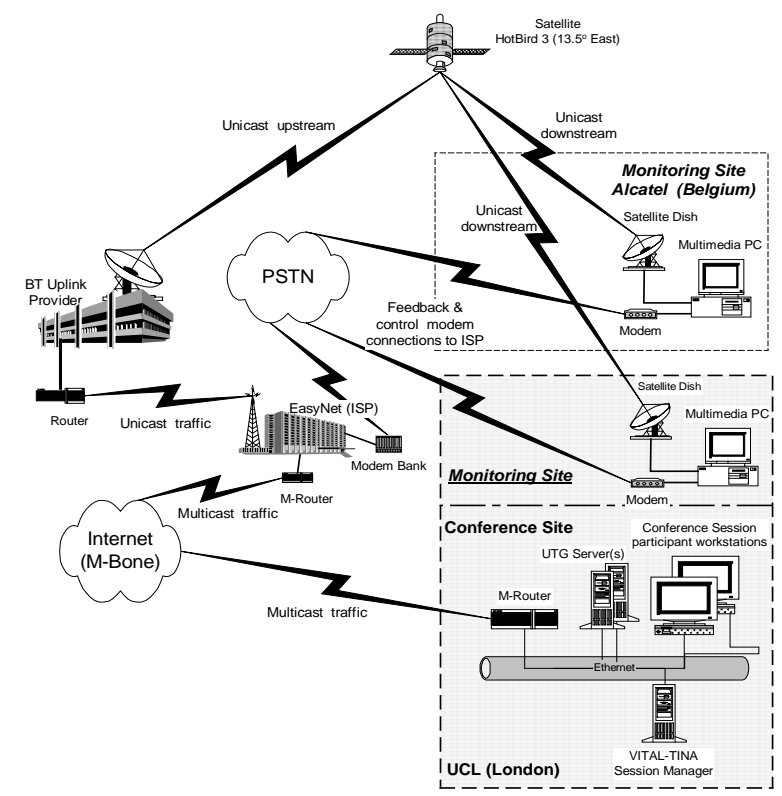

Fig. 9. Schematic of the Experimental Configuration

At the same time the UTG servers would be instructed to establish stream connections with the Satellite PC receivers of both Alcatel and UCL through the connection setup of section 5. It should be noted that the Satellite receivers should not be considered as TINA users, since they the TINA core has no explicit record of their existence. However, it is possible that a user could be accessing his/her local Access Net through a satellite connection. This of course would impose upstream performance limitations since upstreaming for a satellite workstation is only feasible through a modem with the current configuration. Fig. 9 illustrates the DBS connectivity setup configured for the purposes of the demonstration. 


\section{Conclusions and further work}

Investigating the integration of the TINA architecture and Internet-based conferencing services yielded some interesting points essential to the convergence to a TINAInternet synergy. Assuming a TINA legacy, such points provide suitable grounds for TINA in the service provision control of IP-Multicast-based networks.

The impracticality of requiring new components on the Internet-workstations as TINA requires for the TINA terminals, proved to be a valid point; it was shown that Internet-workstations and users were unaware of TINA in the course of the service provision, through the concept of Access Points in the Retailer domain. The TINA components that should normally be instantiated in the workstations of the users could now run at the Access Points. Furthermore, communication between the Internet-workstations and Access Points was based on existing Internet protocols. In this way, TINA was transparent to the Internet-workstations/users.

The transparency offered through the Access Points to the Internet users in the provision of Internet-based services, encourages the implicit brokering of TINA ancillary services such as subscription, accounting or billing management. User access of Internet-based services can be regulated through Access Points by imposing subscription requirements prior any usage. The latter is further regulated by enforcing billing according to subscription characteristics. This is embraced nicely by adopting the notion of Session Control (both at Access and Service level) at the Access Points (Ret domain), interfacing suitably between Internet applications and TINA. Effectively Internet benefits from TINA by using its ancillary service functions. We expect to look into this more seriously in the near future. Having established a viable interface between Internet and TINA we would like to see such ancillary services in use and study their impact on the usage of Internet-based services with respect to QoS and scalability issues.

Our investigation required also end-to-end control of IP-Multicast networks. The added value of the TINA service management (subscription \& accounting management) could only be meaningful if the underlying IP-Multicast network would be centrally controlled so that only authorised users gain access to it. The use of the Unicast<>Multicast Transcoding Gateway (UTG) for this purpose proved most efficient. We enabled the Internet-workstations to gain access to the IP-Multicast network only via the UTG components. In this manner, we could control the user's participation to the network. The UTG components, which were setup and initiated the Access Points, could be easily controlled by the combination of Access Network and core TINA components (AP_TLA and LNC).

We can conclude that TINA can add value to Internet-based services. TINA can control IP-Multicast networks and TINA can provide service management functionality, such as subscription and accounting management, for Internet-based services. One can also infer that the Internet can add value to TINA. We have shown how an Internet Service Provider can use TINA to manage and control Internet services offered to their existing end-users base without any changes to the Internetworkstations of the end-users. 
Apart from this, we had the opportunity to demonstrate that an interworking between the Internet Session Initiation Protocol (SIP) and the TINA call model is achievable. We believe that this kind of interworking could be implemented for other Internet protocols, such as the Session Announcement Protocol (SAP). This is an area that we could investigate in the future that blends nicely with our intents for future work on the interworking between the Internet management of multicast groups and the TINA network resource architecture.

Ultimately, the integrated TINA-Internet system proved quite feasible to apply many of the techniques developed in VITAL for the control and management of applications on the Internet. Such potential, however, renders it necessary to extend the TINA concepts so as to include also an Access Network. If this is done, we have shown how multicast conferencing applications can be run with no TINA-dependent code in the user workstations. It is quite feasible to organise the Access networks so that they carry out the Internet-dependent functions. The interfaces between the components are the true TINA ones, operating in a true DPE over CORBA. The multicast nature of the basic communications makes it straightforward to allow the output streams to go wide-area, by adding the DBS workstations as added participants in the multicast conferences. It would be difficult to make these workstations truly part of the VITAL/Internet scenario, because that would need the CORBA to run across the satellite and return paths. The resulting loss of performance would simply render the operation under such setup to be rather impractical.

\section{Acknowledgements}

Many people contributed to the work here besides the authors. We acknowledge gratefully the support of many people to make the work reported here successful. Our thanks go to the following: the European Commission in funding the VITAL project, the MECCANO project team in supplying their Mbone tools and the UTG, the other VITAL partners in making available their modules as part of the whole integration method, and the Easynet/BT/Eutelsat in making their DBS system available to the demonstration.

\section{References}

1. European ACTS project COIAS (contract number AC363), "COnvergence Internet-ATM Satellite: Annual Project Review Report", CEC Deliverable Number: AC363/COIAS/1120/K/R/P/000/A0, The COIAS Consortium, December 1998. http://www.cs.ucl.ac.uk/staff/jon/coias/review98.html

2. Eutelsat: "High Speed Internet and Data Broadcasting Services: The Benefit of DVB Services on EUTELSAT Satellites", October 1998.

3. Object Management Group, "CORBA Services: Common Object Services Specification", November 1997. http://www.omg.org/

4. Object Management Group, "The Common Object Request Broker Architecture: Architecture and Specification, revision 2.2", Feb 1998. http://www.omg.org/ 
5. H. C. Kim, F. Steegmans, N. Narayanan and J. Rajahalme, "Managing TINA streams that use Internet transport", Proceedings of the Telecommunications Information Networking Architecture Conference, TINA'97, Santiago, Chile, 1997.

6. D. Lewis and T. Tiropanis, "Integrating TINA into an Internet-Based Services Market", Proceedings of the $5^{\text {th }}$ International Conference on Intelligence in Services and Networks, IS\&N '98, Antwerp, Belgium, May 1998.

7. K. Savetz, N. Randall, and Y. Lepage: "Mbone: Multicasting Tomorrow's Internet", ISBN: 1-56884-723-8, IDG Books Worldwide, Inc., July 1996

8. R. Bennett, P. T. Kirstein, "Technical Innovations deployed by the MERCI Project ", Proc Networkshop 25, Belfast, March 1997, pages 181-189

9. V. Hardman, M. A. Sasse \& I. Kouvelas, "Successful Multiparty Audio Communication over the Internet", Communications of the ACM, Vol. 41(5), 1998.

10. M. Handley: "SAP: Session Announcement Protocol", Internet-draft, version 5, IETF, April 1998.

11. G. Montasser, "User Guide for SDR v2.5", Version 15 (DRAFT), UCL-CS, September 1998. http://www-mice.cs.ucl.ac.uk/multimedia/software/

12. M. Handley, H. Schulzrinne and E. Schooler: "SIP: Session Initiation Protocol", Internetdraft, version 7, IETF, July 1998.

13. C. Semeria, T. Maufer: "Introduction to IP Multicast Routing", Internet-draft, IETF, January 1997.

14. TINA-C: "Network Resource Architecture", version 3.0, February 1997.

15. TINA-C: "Overall Concepts and Principles of TINA", February 1995.

16. TINA-C: "Service Architecture", version 5.0, June 1997.

17. P. T. Kirstein and J. Yang: "Accessing MBone Sessions over Point-to-point Connection", Private Communication, UCL-CS, 1997.

18. L. Clark, " User Guide for UTG v1.3", Version 2 (DRAFT), UCL-CS, 1997. http://wwwmice.cs.ucl.ac.uk/multimedia/software/

19. S. McCanne, and V. Jacobson, "vic: A Flexible Framework for Packet Video", ACM Multimedia, July 1995.

20. European ACTS project VITAL (contract number AC003), "ODTA Validation Second Trial, parts (A, B and C)", CEC Deliverable Number: AC003/SES/WP2/DS/P/D10/b1, The VITAL Consortium, October 1997. http://www.mari.co.uk/vital/

21. European ACTS project VITAL (contract number AC003), "ODTA Validation Third Trial, parts (A, B and C)", CEC Deliverable Number: AC003/SES/WP2/DS/P/D13/b1, The VITAL Consortium, October 1998.

22. L. Clark, "User Guide for WB v1.59", Version 1 (DRAFT), UCL-CS, September 1998. http://www-mice.cs.ucl.ac.uk/multimedia/software/ 